\title{
Long non-coding RNA XIST promotes cell proliferation and migration through targeting miR-133a in bladder cancer
}

\author{
KEQIN ZHOU, JINRUI YANG, XURUI LI and WENJIE CHEN
}

Department of Urology, The Second Xiangya Hospital, Central South University, Changsha, Hunan 410011, P.R. China

Received November 17, 2018; Accepted May 16, 2019

DOI: $10.3892 /$ etm.2019.7960

\begin{abstract}
The long non-coding RNA (lncRNA) X inactive specific transcript (XIST) has recently been reported to promote the malignant progression of bladder cancer through regulating several microRNAs (miRs), including miR-124, miR-139-5p and miR-200c. However, whether other miRs are also involved in this process has remained to be determined. The present study demonstrated that XIST was significantly upregulated in bladder cancer tissues compared with that in adjacent normal tissues. Furthermore, its expression was reduced in several common bladder cancer cell lines. High expression of XIST was significantly associated with tumour progression and poor prognosis of patients with bladder cancer. An in vitro experiment indicated that knockdown of XIST significantly reduced the proliferation and migration of bladder cancer cells. A luciferase assay suggested that XIST binds to its predicted binding site in miR-133a. In addition, it was identified that miR-133a was significantly downregulated in bladder cancer, and its expression levels were inversely correlated with those of XIST in bladder cancer tissues. Furthermore, loss- and gain-of-function experiments indicated that miR-133a acted as a downstream effector in XIST-mediated bladder cancer cell proliferation and migration. In conclusion, the present study demonstrates that XIST promotes bladder cancer cell proliferation and migration via targeting miR-133a and thus suggests that XIST may be used as a potential therapeutic target for bladder cancer.
\end{abstract}

\section{Introduction}

Bladder cancer is one of the most common cancer types of the urological tract, with increasing incidence in recent years (1-3). Despite systemic therapy for bladder cancer, the clinical outcome is modest, mainly due to metastasis, recurrence and

Correspondence to: Mr. Keqin Zhou, Department of Urology, The Second Xiangya Hospital, Central South University, 139 Renmin Road, Changsha, Hunan 410011, P.R. China

E-mail: zhoukeqin0713@163.com

Key words: long non-coding RNA, X inactive specific transcript, bladder cancer, microRNA drug resistance (4). During the development and progression of bladder cancer, numerous tumour suppressors and oncogenes, including certain microRNAs (miRNAs/miRs) and long non-coding RNAs (lncRNAs), have been determined to be deregulated $(1,5,6)$. Gaining insight into the molecular mechanisms of bladder cancer is beneficial for developing novel biomarkers and therapeutic targets for its diagnosis and treatment (7).

lncRNAs are a class of non-protein-coding transcripts with lengths of $>200$ base pairs and are involved in the pathological process of numerous human diseases through regulating processes including cell proliferation, apoptosis, migration and angiogenesis (8-12). Accumulating evidence has indicated that numerous lncRNAs have either oncogenic or tumour-suppressive roles in bladder cancer $(13,14)$. For instance, the lncRNA activated by transforming growth factor- $\beta$ (TGF- $\beta$ ) is upregulated in bladdercancer and promotes the proliferation, migration and invasion of bladder cancer cells by inhibiting the expression of miR-126 (15). Wang et al (16) determined a negative correlation between growth arrest specific 5 (GAS5) levels and clinical stage in bladder cancer, and overexpression of GAS5 reduced bladder cancer cell viability and induced apoptosis.

$\mathrm{X}$ inactivation is an early developmental process in mammalian females that transcriptionally silences one of the pairs of $\mathrm{X}$ chromosomes (17). The lncRNA X inactive specific transcript (XIST) is required for $\mathrm{X}$ inactivation during development (18). Recently, XIST has been reported to be frequently upregulated in various human cancer types and to function as an oncogene $(19,20)$. For instance, it may accelerate cervical cancer progression via upregulating Fus through competitively binding with miR-200a (20). In addition, XIST may promote gastric cancer progression through TGF- $\beta 1$ via targeting miR-185 (19).

The oncogenic role of XIST in bladder cancer has also been reported $(21,22)$. For instance, Xu et al (21) suggested that the lncRNA XIST inhibits the stemness properties and tumourigenicity of bladder cancer cells through inhibiting the expression of miR-200c. In addition, XIST promotes cell growth and metastasis through regulating the miR-139-5p-mediated Wnt/ $\beta$-catenin signalling pathway in bladder cancer (22). Recently, Wei et al (23) reported that XIST promoted pancreatic cancer cell proliferation through regulating the expression of miR-133a and epidermal growth factor receptor (EGFR). However, whether miR-133a is also involved in XIST-mediated bladder cancer has remained elusive. 
In the present study, the clinical significance of XIST in bladder cancer was assessed and in addition, the regulatory mechanism of XIST in bladder cancer cell proliferation and migration were explored.

\section{Materials and methods}

Clinical tissue samples. Bladder cancer tissues and adjacent tissues were collected from 52 primary bladder cancer patients at the Second Xiangya Hospital (Changsha, China) between March 2011 and April 2013. The clinical characteristics of the patients are summarized in Table I and included 32 male and 20 female patients, aged between 42-75 years. Prior to surgery, none of the cancer patients had been treated by chemotherapy or radiotherapy. The tissues were rapidly frozen in liquid nitrogen and stored until use.

Cell culture and transfection. The SV-HUC-1 normal urinary tract epithelial cell line and four common bladder cancer cell lines, T24, 253J, RT112 and HT-1376, were obtained from the Cell Bank of the Chinese Academy of Sciences. These cell lines were cultured in Dulbecco's modified Eagle's medium (DMEM; Thermo Fisher Scientific, Inc.) supplemented with $10 \%$ foetal bovine serum (Thermo Fisher Scientific, Inc.) at $37^{\circ} \mathrm{C}$ with $5 \% \mathrm{CO}_{2}$. For cell transfection, 253J and RT112 cells were transfected with $100 \mathrm{nM}$ XIST small interfering (si)RNA1 (5'-GCAAAUGAAAGCUACCAAU-3'; Thermo Fisher Scientific, Inc.), XIST siRNA2 (5'-GCACAAUAU CUUUGAACUA-3'; Thermo Fisher Scientific, Inc.) or 100 nM negative control (NC) siRNA (cat. no. 4459405; Thermo Fisher Scientific, Inc.) or co-transfected with $100 \mathrm{nM}$ NC siRNA and 100 nM NC inhibitor (cat. no. 4464076; Thermo Fisher Scientific, Inc.), or $100 \mathrm{nM} \mathrm{NC} \mathrm{siRNA} \mathrm{and}$ 100 nM miR-133a inhibitor (cat. no. 4464084; Thermo Fisher Scientific, Inc.), or 100 nM XIST siRNA and 100 nM miR-133a inhibitor, or $100 \mathrm{nM}$ XIST siRNA and $100 \mathrm{nM}$ NC inhibitor using Lipofectamine 2000 (Thermo Fisher Scientific, Inc.) according to the manufacturer's protocols. At $48 \mathrm{~h}$ after transfection, the cells were harvested and used for the subsequent assays.

Reverse transcription-quantitative $(R T-q) P C R$. Total RNA was isolated from tissues and cell lines using TRIzol reagent (Thermo Fisher Scientific, Inc.) and RT was performed by processing 500 ng RNA with the First-Stand cDNA Synthesis Kit (Tiangen Biotech Co., Ltd) according to the manufacturer's protocols. qPCR analysis was performed using SYBR Green PCR Master Mix (Takara Bio, Inc.) according to the manufacturer's protocols. The reaction conditions were $95^{\circ} \mathrm{C}$ for $3 \mathrm{~min}$ followed by 40 cycles of $95^{\circ} \mathrm{C}$ for $15 \mathrm{sec}$ and $60^{\circ} \mathrm{C}$ for $15 \mathrm{sec}$. Relative gene expression was calculated using the $2^{-\Delta \Delta \mathrm{Ca}}$ method (24). GAPDH and U6 were used as internal references. The primers utilized in PCR were as follows: XIST forward, 5'-ACGCTGCATGTGTCCTTAG-3' and reverse, 5'-GAGCCT CTTATAGCTGTTTG-3'; GAPDH forward, 5'-CTGGGC TACACTGAGCACC-3' and reverse, 5'-AAGTGGTCGTTG AGGGCAATG-3'; miR-133a forward, 5'-TTTGGTCCCCTT CAACCAGCTG-3' and reverse, 5'-TAAACCAAGGTAAAA TGGTCGA-3'; U6 forward, 5'-CTCGCTTCGGCAGCACA-3' and reverse, 5'-AACGCTTCACGAATTTGCGT-3'.
Cell Counting Kit-8 (CCK-8) assay. At 48 h after transfection, 253J and RT112 cells were seeded onto 96-well plates at 5,000 cells per well. After incubation at $37^{\circ} \mathrm{C}$ with $5 \% \mathrm{CO}_{2}$ for $0,24,48$ and $72 \mathrm{~h}$, cell proliferation was measured using the CCK-8 assay (Thermo Fisher Scientific, Inc.) according to the manufacturer's protocols. Absorbance was detected at an optical density of $450 \mathrm{~nm}$ by a spectrophotometer (BioRad Laboratories, Inc.).

Wound healing assay. To study cell migration, transfected 253J and RT112 cells were seeded into 6-well plates. After culturing at $37^{\circ} \mathrm{C}$ with $5 \% \mathrm{CO}_{2}$ for $24 \mathrm{~h}$, a line was scraped into the monolayer with a $200-\mu 1$ micropipette tip. The cells were washed twice with PBS and cultured in serum-free DMEM for $24 \mathrm{~h}$. Images of the wounded area at 0 and $24 \mathrm{~h}$ were captured under a light microscope (CX22; Olympus Corporation).

Bioinformatics analysis and luciferase reporter gene assay. The potential targeting miRs of the lncRNA XIST were predicted using RNAhybrid 2.12 (http://bibiserv.techfak. uni-bielefeld.de/rnahybrid/) (25). XIST sequences containing the wild-type (WT) or mutant-type (MT) miR-133a binding sites were subcloned into the pmiR-GLo luciferase reporter vector (Promega Corp.). Lipofectamine 2000 was then used to transfect 253J and RT112 cells with miR-NC or miR-133a mimics together with the WT- or MT-XIST reporter plasmid according to the manufacturer's protocols. At $48 \mathrm{~h}$ after transfection, a Dual Luciferase Reporter Assay System (Promega Corp.) was used to determine luciferase activities according to the manufacturer's protocols.

Statistical analysis. Values are expressed as the mean \pm standard deviation. SPSS 20.0 software (IBM Corp.) was used for statistical analysis. An unpaired two-tailed Student's t-test was used to compare the differences between two groups. One-way analysis of variance followed by Tukey's post-hoc test was used for multiple comparisons. The association between XIST expression and the clinical features of bladder cancer patients was analysed using the chi-square test. For survival analysis, Kaplan-Meier survival curves were drawn and the log-rank test was applied. The Spearman rank correlation was used to analyse the correlation between the expression of XIST and miR-133a in bladder cancer tissues. $\mathrm{P}<0.05$ was considered to indicate statistical significance.

\section{Results}

Upregulation of XIST is associated with tumour progression and poor prognosis in bladder cancer. To explore the function of XIST in bladder cancer, its expression in the normal urinary tract epithelial cell line SV-HUC-1 and four common bladder cancer cell lines, T24, 253J, RT112 and HT-1376, was first examined. The results of the RT-qPCR analysis suggested that the expression levels of XIST were significantly increased in bladder cancer cells compared with those in SV-HUC-1 cells (Fig. 1A). To confirm these results, 52 paired bladder cancer tissues and adjacent normal tissues were collected and assessed for expression of XIST. The results revealed that XIST was also significantly upregulated in bladder cancer tissues vs. adjacent normal tissues (Fig. 1B). Furthermore, high XIST 
Table I. Association between XIST expression and clinicopathological characteristics in bladder cancer.

\begin{tabular}{|c|c|c|c|c|}
\hline \multirow[b]{2}{*}{ Characteristic } & \multirow[b]{2}{*}{ Cases $(\mathrm{n}=52)$} & \multicolumn{2}{|c|}{ XIST expression } & \multirow[b]{2}{*}{ P-value } \\
\hline & & $\operatorname{High}(n=19)$ & Low $(n=33)$ & \\
\hline Age (years) & & & & 0.546 \\
\hline$<55$ & 18 & 8 & 10 & \\
\hline$\geq 55$ & 34 & 11 & 23 & \\
\hline Sex & & & & 0.558 \\
\hline Male & 32 & 13 & 19 & \\
\hline Female & 20 & 6 & 14 & \\
\hline Degree of differentiation & & & & 0.095 \\
\hline Well/moderate & 40 & 12 & 28 & \\
\hline Poor & 12 & 7 & 5 & \\
\hline Lymph node metastasis & & & & 0.001 \\
\hline No & 33 & 6 & 27 & \\
\hline Yes & 19 & 13 & 6 & \\
\hline Distant metastasis & & & & 0.001 \\
\hline No & 46 & 13 & 33 & \\
\hline Yes & 6 & 6 & 0 & \\
\hline TNM stage & & & & 0.001 \\
\hline $\mathrm{I} / \mathrm{II}$ & 28 & 4 & 24 & \\
\hline III/IV & 24 & 15 & 9 & \\
\hline
\end{tabular}

TNM,tumour-nodes-metastasis; XIST, $\mathrm{X}$ inactive specific transcript.
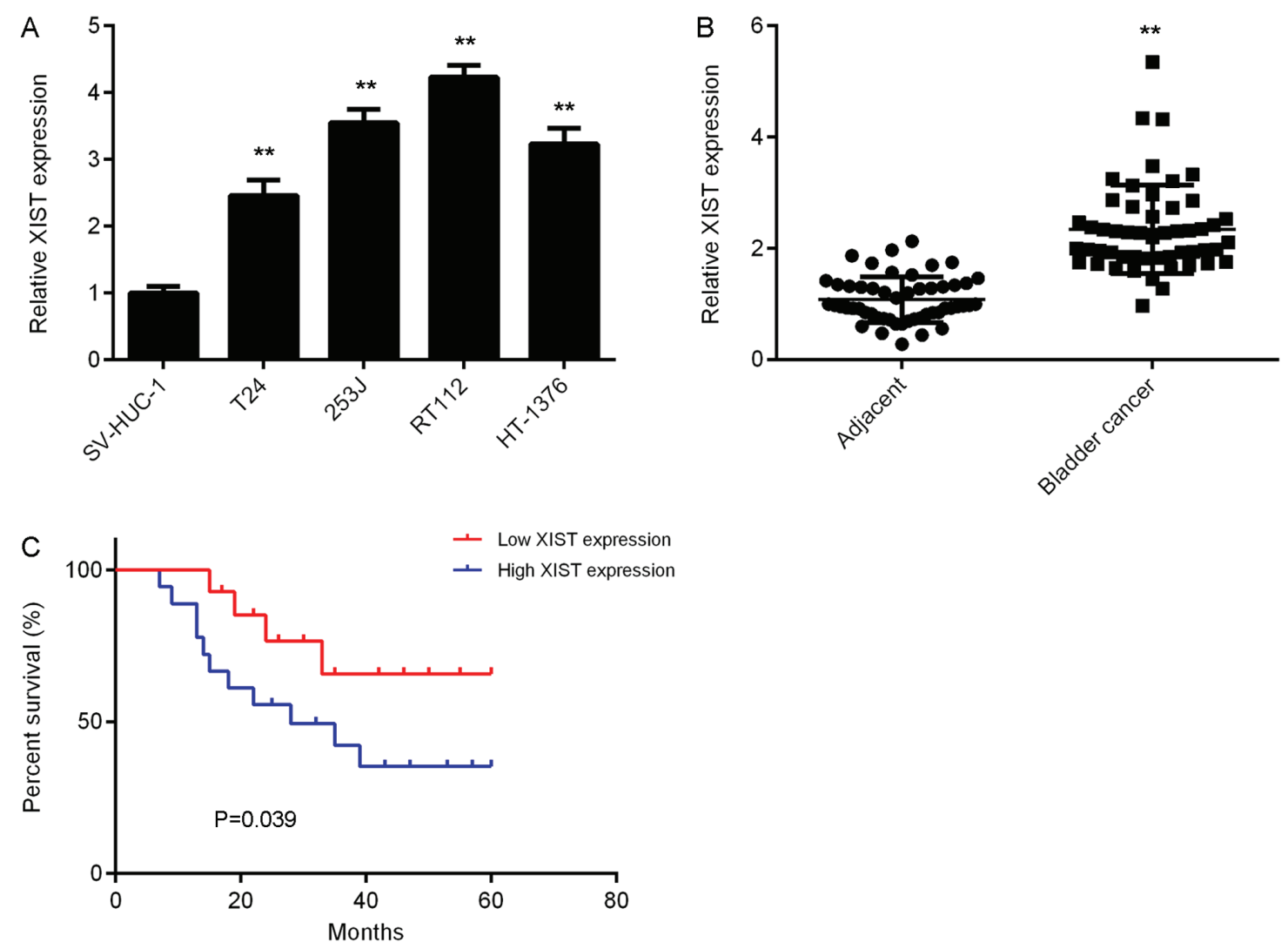

Figure 1. Upregulation of XIST in bladder cancer. (A) qPCR was used to examine XIST expression in the normal urinary tract epithelial cell line SV-HUC-1 and 4 bladder cancer cell lines. ${ }^{* *} \mathrm{P}<0.01$ vs. SV-HUC-1. (B) qPCR was used to examine the XIST expression in 52 paired bladder cancer tissues and adjacent normal tissues. ${ }^{* *} \mathrm{P}<0.01$ vs. Adjacent. (C) High XIST expression was significantly associated with a shorter survival time of bladder cancer patients. XIST, X inactive specific transcript; qPCR, quantitative polymerase chain reaction. 

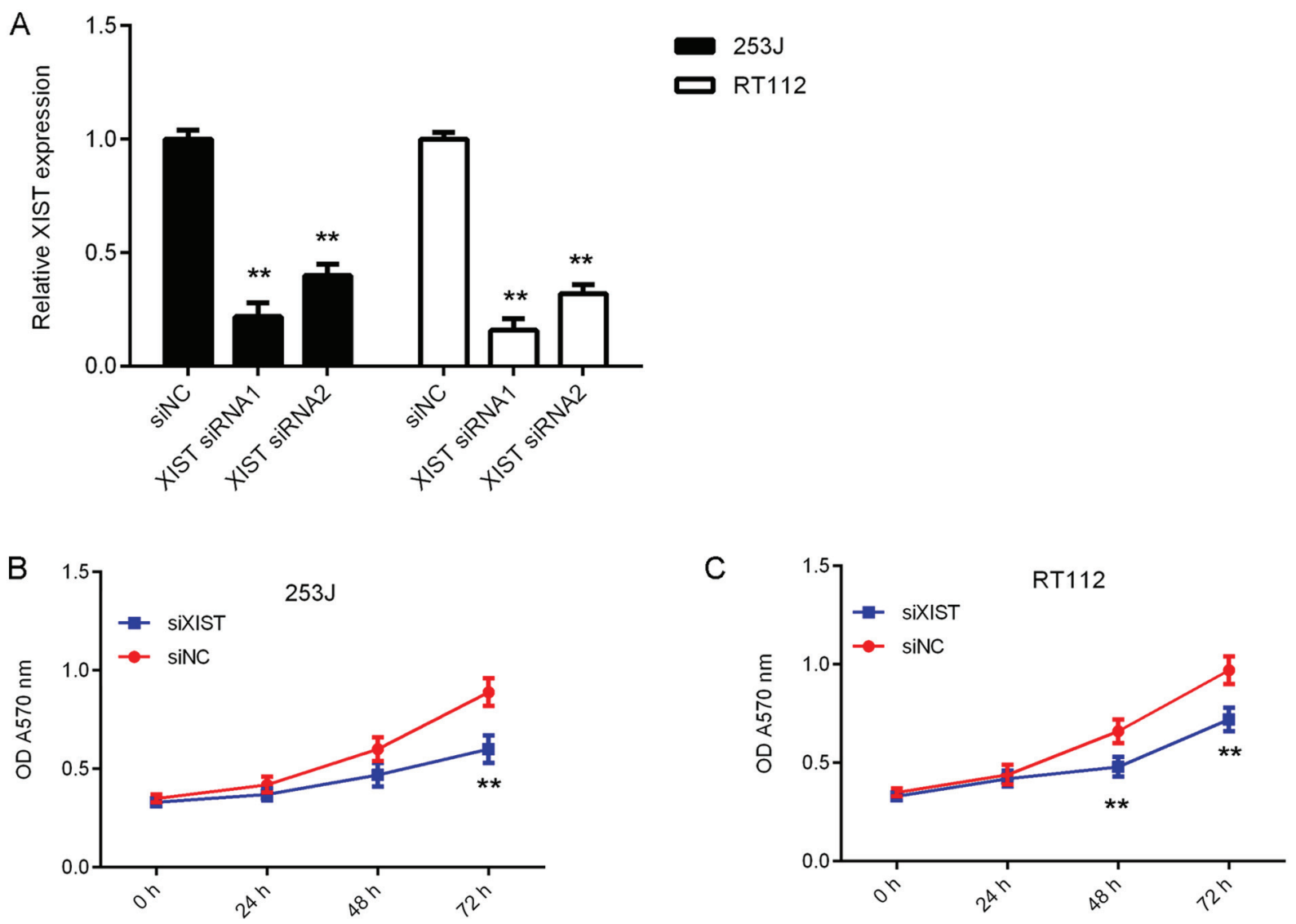

D 253J siNC siXIST
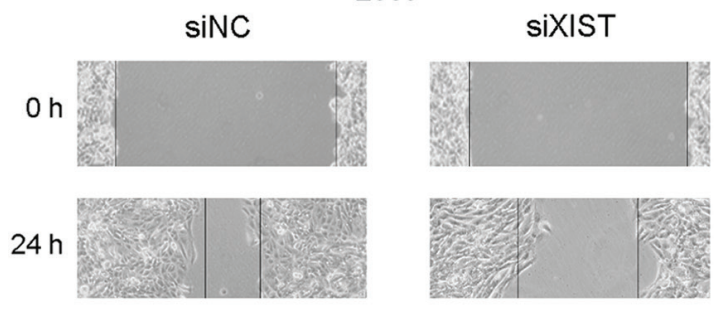

$\mathrm{E}$ 253J RT112
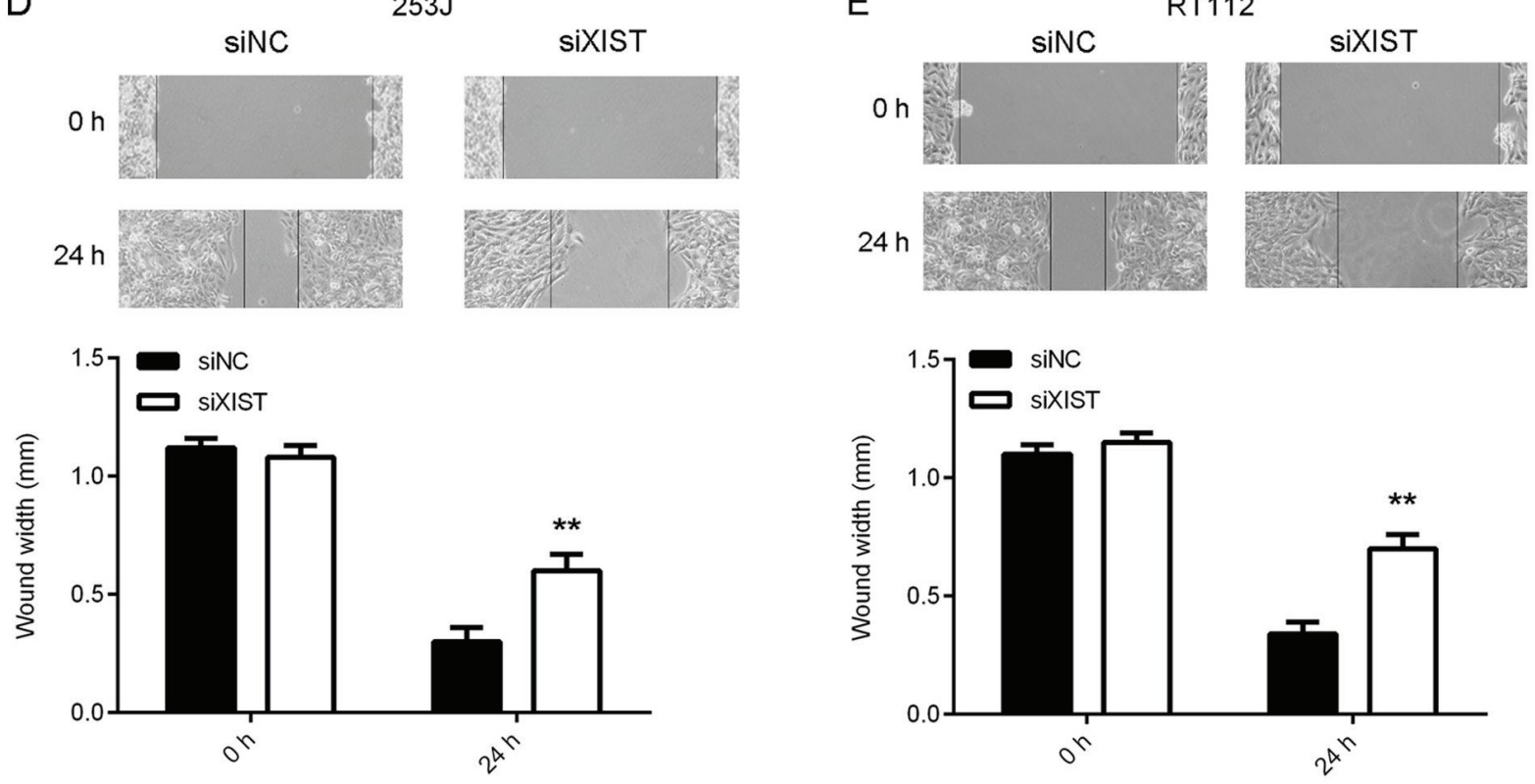

Figure 2. Inhibition of XIST suppresses bladder cancer cell proliferation or migration. 253J and RT112 cells were transfected with NC siRNA, XIST siRNA1 or XIST siRNA2. (A) qPCR was used to examine the expression of XIST. (B and C) A Cell Counting Kit-8 assay and (D and E) a wound healing assay (magnification, $\mathrm{x} 40$ ) were used to assess the effects of the treatments on cell proliferation and migration, respectively. ${ }^{* *} \mathrm{P}<0.01 \mathrm{vs}$. siNC. XIST, $\mathrm{X}$ inactive specific transcript; siRNA, small interfering RNA; siNC, negative control siRNA; siXIST, siRNA targeting XIST; OD, optical density.

expression was significantly associated with the presence of metastasis, advanced tumour-nodes-metastasis (TNM) stage and a shorter survival time of bladder cancer patients (Table I, Fig. 1C). It was therefore indicated that upregulation of XIST is associated with tumour progression and poor prognosis in bladder cancer.
Inhibition of XIST suppresses bladder cancer cell proliferation and migration. In the present study, 253J and RT112 cells were subjected to in vitro loss-of-function experiments to further reveal the function of XIST in bladder cancer. 253J and RT112 cells were transfected with NC siRNA, or XIST siRNA1 or siRNA2. After transfection, the expression levels of XIST 
A

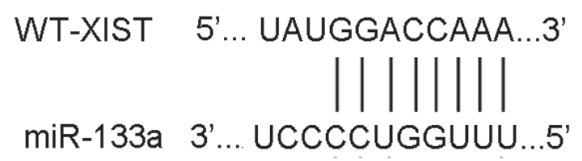

MT-XIST 5 '... UAUCCUGGUUU...3'
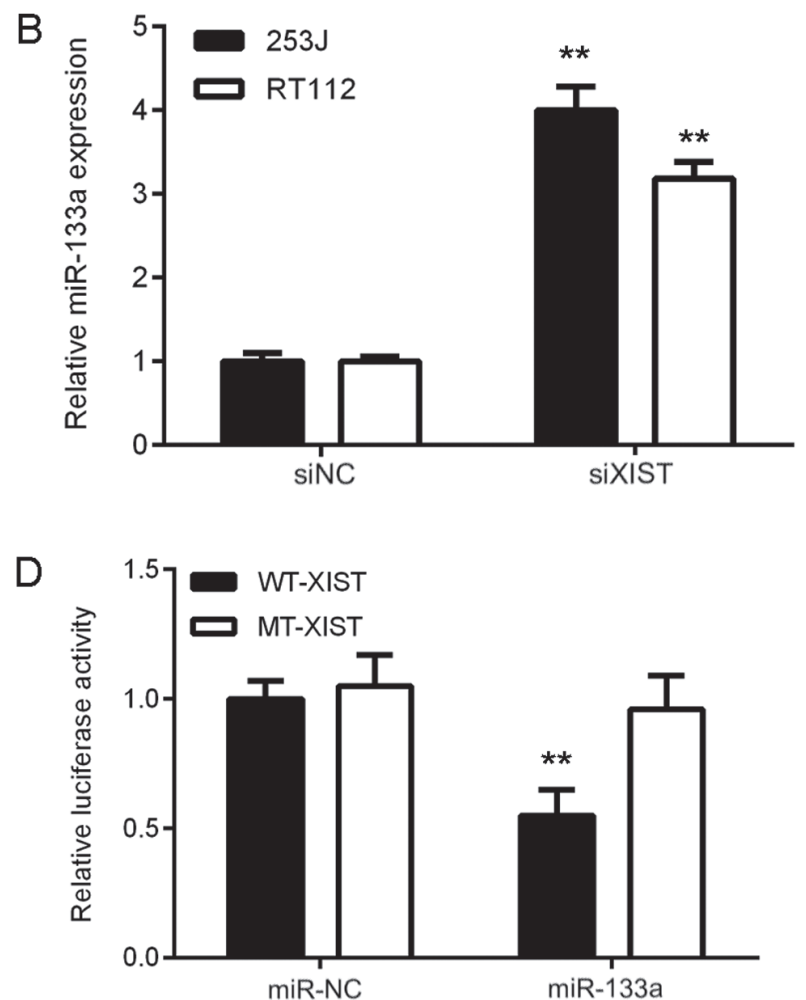

Figure 3. XIST directly targets miR-133a in bladder cancer cells. (A) Bioinformatics analysis suggested that miR-133a has putative XIST binding sites, and luciferase reporter plasmids containing the WT and MT miR-133a binding sites in XIST were generated. (B) Knockdown of XIST significantly promoted miR-133a expression in $253 \mathrm{~J}$ and RT112 cells. ${ }^{* *} \mathrm{P}<0.01$ vs. siNC. (C and D) Luciferase reporter gene assay suggested that transfection with miR-133a mimics decreased the luciferase activity of WT XIST reporter plasmid, while not affecting the luciferase activity of MT XIST reporter plasmid in 253J and RT112 cells. ${ }^{* *} \mathrm{P}<0.01$ vs. miR-NC. XIST, $\mathrm{X}$ inactive specific transcript; miR, microRNA; miR-NC, miR mimics negative control; WT, wild type; MT, mutant type; siRNA, small interfering RNA; siNC, negative control siRNA; siXIST, siRNA targeting XIST.

were significantly reduced in the XIST siRNA1 and siRNA2 groups compared with those in the siNC group (Fig. 2A). As XIST siRNA1 exhibited a better suppressive effect on XIST expression, it was used in the subsequent experiments. The results of the CCK- 8 assay indicated that silencing of XIST expression caused a reduction in bladder cancer cell proliferation (Fig. 2B and C). Furthermore, as indicated in Fig. 2D and E, the migration of 253J and RT112 cells was also inhibited after knockdown of XIST. Taken together, these results suggest that the inhibition of XIST suppresses bladder cancer growth and metastasis.

XIST directly targets miR-133a in bladder cancer cells. Next, a Bioinformatics analysis was performed to predict potential XIST-miR interactions. The results indicated that miR-133a had a potential binding site in XIST (Fig. 3A). Furthermore, knockdown of XIST expression significantly promoted miR-133a expression in bladder cancer cells (Fig. 3B). To verify the predicted direct binding interaction, a luciferase reporter gene assay was then performed. As indicated in Fig. 3C and D, miR-133a mimics significantly inhibited the luciferase activity of the XIST-WT luciferase reporter gene plasmid but had no effect on that of the XIST-MT plasmid in bladder cancer cells, suggesting that XIST directly targets miR-133a in bladder cancer cells.

Downregulation of miR-133a in bladder cancer. The expression levels of miR-133a in bladder cancer were then assessed. As presented in Fig. 4A, miR-133a was significantly downregulated in bladder cancer cells compared with that in SV-HUC-1 cells. Furthermore, the expression levels of miR-133a were significantly downregulated in bladder cancer tissues compared with those in adjacent normal tissues (Fig. 4B). Further investigation revealed an inverse correlation between miR-133a and XIST expression in bladder cancer tissues (Fig. 4C), suggesting that the reduced expression of miR-133a may be due to the increased expression of XIST in bladder cancer.

miR-133a acts as a downstream effector in XIST-mediated bladder cancer cell proliferation and migration. Next, it was assessed whether miR-133a acts as a downstream effector in XIST-mediated bladder cancer cell proliferation and migration. 253J and RT112 cells were co-transfected with NC siRNA and NC inhibitor (siNC+anti-NC), NC siRNA and miR-133a inhibitor (si-NC+anti-miR-133a), XIST siRNA and NC inhibitor (siXIST+anti-NC), or XIST siRNA and miR-133a inhibitor (siXIST+anti-miR-133a). After transfection, the miR-133a levels were significantly reduced in the si-NC+anti-miR-133a group, but significantly increased in the siXIST+anti-NC group, when compared with those in the siNC+anti-NC group (Fig. 5A and B). However, no significant difference in miR-133a expression was observed between the siNC+anti-NC and siXIST+anti-miR-133a groups. CCK-8 and wound healing assays were then performed to assess the effects of the above treatments on cell proliferation and migration. As indicated in 

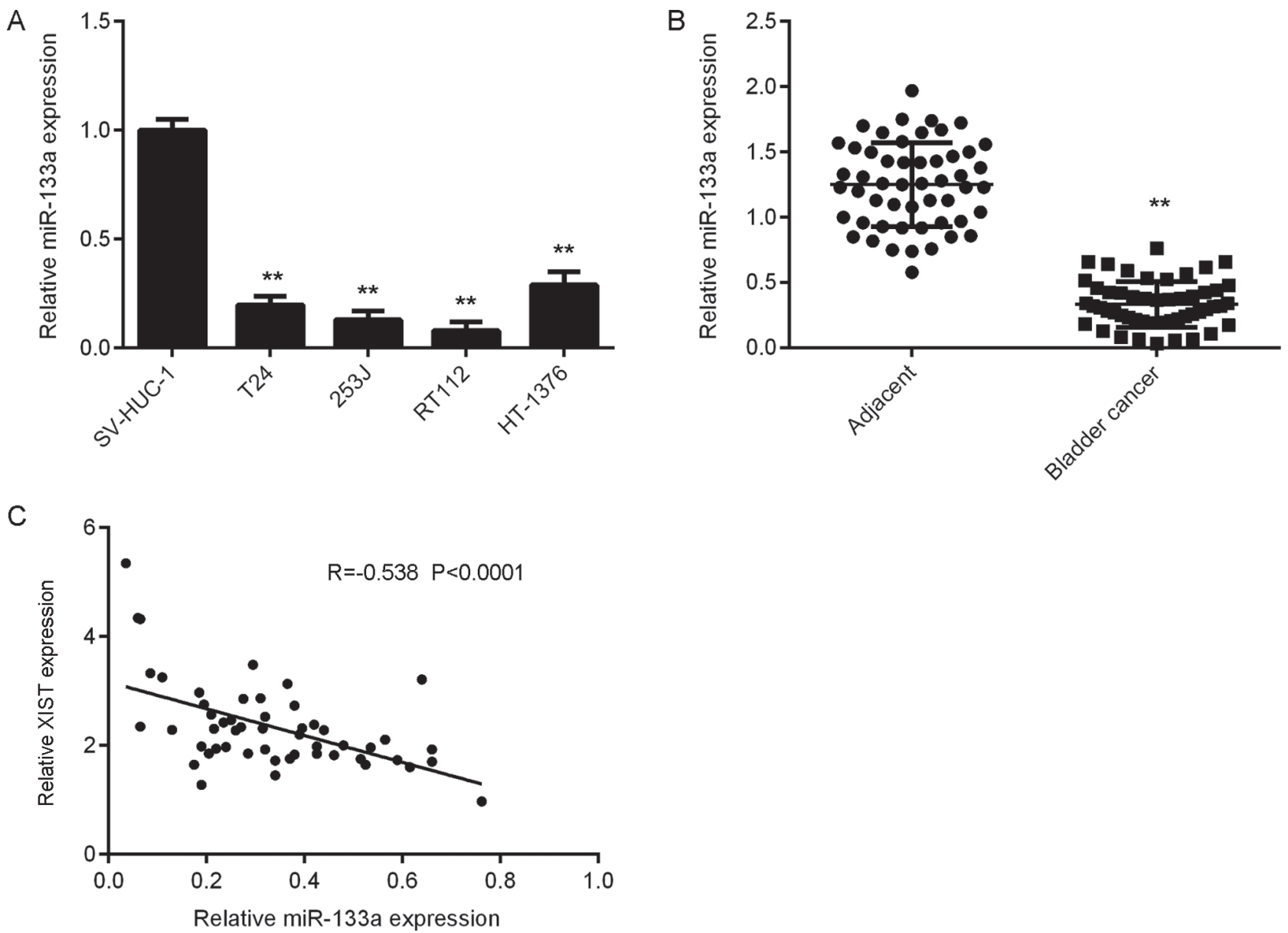

Figure 4. miR-133a is downregulated in bladder cancer tissues and cell lines. (A) RT-qPCR was used to examine the miR-133a expression in a normal urinary tract epithelial cell line SV-HUC-1 and 4 bladder cancer cell lines. ${ }^{* *} \mathrm{P}<0.01$ vs. SV-HUC-1. (B) RT-qPCR was used to examine the miR-133a expression in 52 paired bladder cancer tissues and adjacent normal tissues. ${ }^{* *} \mathrm{P}<0.01$ vs. Adjacent. (C) Inverse correlation between miR-133a and XIST expression in bladder cancer tissues. XIST, $\mathrm{X}$ inactive specific transcript; miR, microRNA; RT-qPCR, reverse-transcription quantitative PCR.

Fig. 5C-F, the proliferation and migration of bladder cancer cells were significantly increased in the siNC+anti-miR-133a group, but significantly decreased in the siXIST+anti-NC group, when compared with those in the siNC+anti-NC group. In addition, no significant difference was identified between the siNC+anti-NC and siXIST+anti-miR-133a groups (Fig. 5C-F). These results indicate that inhibition of miR-133a impairs the suppressive effects of XIST downregulation on the proliferation and migration of bladder cancer cells. Therefore, miR-133a acts as a downstream effector in XIST-mediated bladder cancer cell proliferation and migration.

\section{Discussion}

It has been demonstrated that IncRNAs affect the expression of a large number of genes and miRNAs at the transcriptional and post-transcriptional levels, through which they regulate various cellular biological processes, including cell proliferation, cell cycle progression, apoptosis, migration and tumourigenesis $(10,11,21,26,27)$. However, the regulatory mechanisms of the lncRNA XIST during bladder cancer progression have remained to be fully elucidated. In the present study, it was indicated that XIST was significantly upregulated in bladder cancer tissues vs. adjacent normal tissues. Furthermore, its expression was also reduced in several common bladder cancer cell lines. High expression of XIST was significantly associated with tumour progression and poor prognosis of patients with bladder cancer. The in vitro experiments suggested that knockdown of XIST significantly reduced the proliferation and migration of bladder cancer cells. Furthermore, a luciferase assay confirmed that XIST directly binds with miR-133a at the predicted binding site. It was also determined that miR-133a was significantly downregulated in bladder cancer, and its expression levels were inversely correlated with XIST expression levels in bladder cancer tissues. In addition, loss- and gain-of-function experiments suggested that miR-133a acts as a downstream effector in XIST-mediated bladder cancer cell proliferation and migration.

In recent years, the IncRNA XIST has been reported to be frequently upregulated in certain common types of cancer, and its upregulation is associated with tumour progression and poor prognosis of cancer patients (28-30). For instance, Yang et al (31) indicated that the expression of XIST was significantly increased in osteosarcoma tissues and cell lines, and negatively correlated with clinical prognosis. Sun et al (32) reported that XIST was markedly upregulated in pancreatic cancer, and its overexpression significantly promoted cancer cell proliferation, migration and invasion. In the present study, 
A
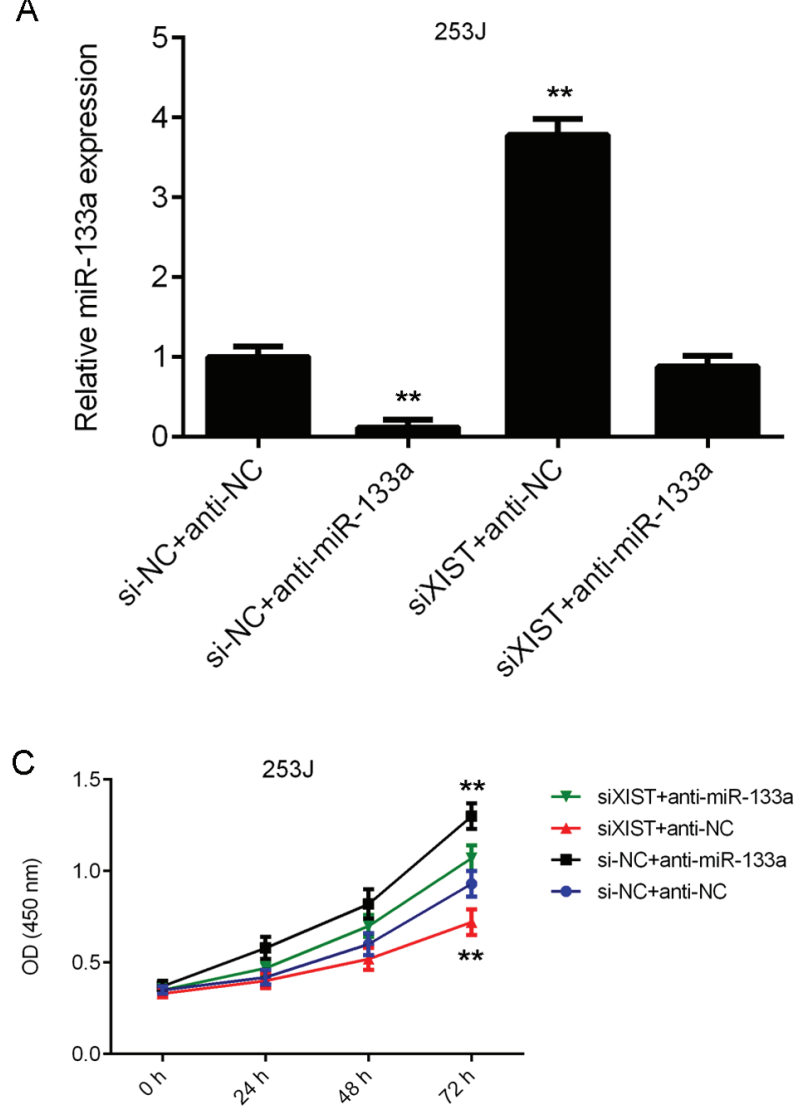

$\mathrm{E}$

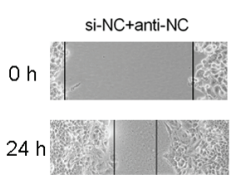

$253 \mathrm{~J}$
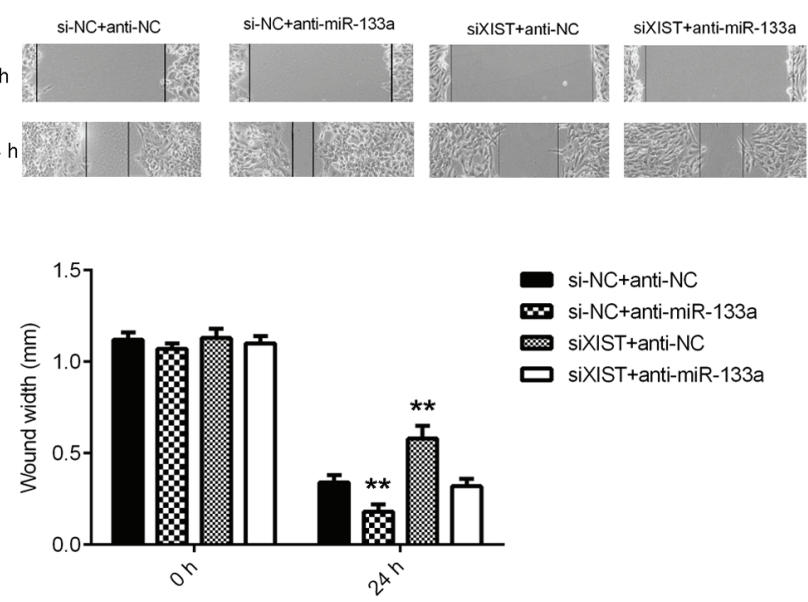

B
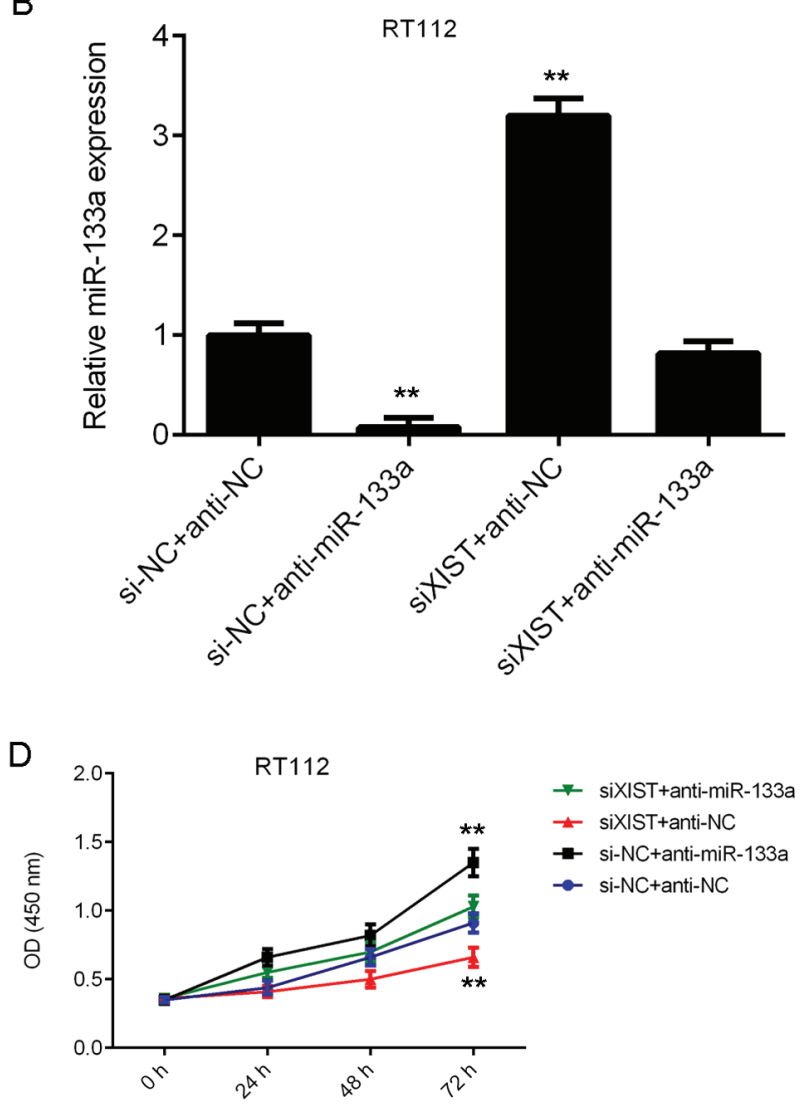

$\mathrm{F}$
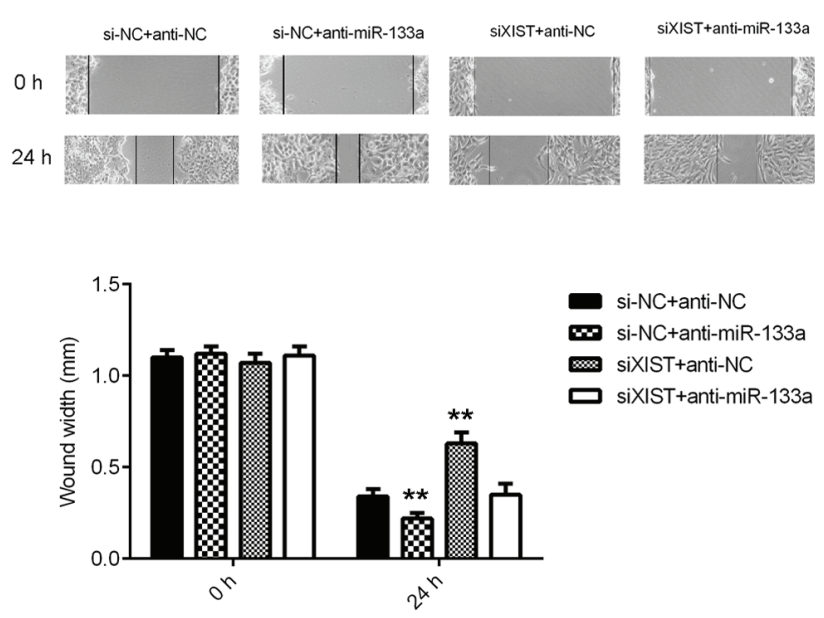

Figure 5. miR-133a acts as a downstream effector in XIST-mediated bladder cancer cell proliferation and migration. 253J and RT112 cells were co-transfected with NC siRNA and NC inhibitor, NC siRNA and miR-133a inhibitor, XIST siRNA and NC inhibitor or XIST siRNA and miR-133a inhibitor, respectively. (A and B) The expression of XIST was examined using reverse-transcription quantitative PCR. (C and D) A Cell Counting Kit-8 assay and (E and F) a wound healing assay (magnification, $\mathrm{x} 40$ ) were performed to assess cell proliferation and migration, respectively. ${ }^{* *} \mathrm{P}<0.01$ vs. si-NC+anti-NC. XIST, $\mathrm{X}$ inactive specific transcript; miR, microRNA; anti-miR-133a, miR-133a inhibitor; anti-NC, miR inhibitor control, siRNA, small interfering RNA; siNC, negative control siRNA; siXIST, siRNA targeting XIST; OD, optical density.

increased expression of XIST in bladder cancer tissues and cell lines was detected and high expression of XIST was associated with positive metastasis, advanced TNM stage and shorter survival time of bladder cancer patients. Similarly, Xiong et al (33) also reported that XIST was upregulated in bladder cancer tissues and that higher XIST expression was associated with advanced TNM stage of bladder cancer. In addition, Hu et al (22) reported that XIST was significantly upregulated in bladder cancer tissues and cell lines, and correlated with poor prognosis of bladder cancer patients. Taken together, the results of previous and the present study suggest that the lncRNA XIST has a key role during bladder cancer progression and may thus serve as a potential therapeutic target.

To further study the function of XIST in tumour growth and metastasis, bladder cancer cells were transfected with 
XIST siRNA to knock down its expression, and CCK-8 and wound healing assays suggested that the downregulation of XIST caused a significant reduction in bladder cancer cell proliferation and migration. These results suggest that the IncRNA XIST may have a promoting role during bladder cancer growth and metastasis. Consistent with these results, Xiong et al (33) also reported that XIST knockdown inhibited the proliferation, invasion and migration of bladder cancer cells. In addition, Hu et al (22) indicated that silencing of XIST expression significantly reduced bladder cancer cell growth and metastasis in vitro and tumour growth in vivo. However, the molecular mechanisms of the effect of XIST on bladder cancer progression have remained to be fully elucidated.

The present study focused on downstream miRNAs of XIST, as numerous miRNAs have been reported to act as tumour suppressors and oncogenes in bladder cancer $(5,34)$. Bioinformatics analysis predicted that XIST and miR-133a contain mutual binding sites. In fact, several previous studies have reported that miR-133a functions as a tumour suppressor in bladder cancer. For instance, miR-133a was demonstrated to induce bladder cancer cell apoptosis through directly targeting glutathione S-transferase pi 1 (35). Zhou et al (36) reported that miR-133 inhibited the proliferation, migration and invasion of bladder cancer cells by targeting EGFR and its downstream effector proteins. In the present study, it was demonstrated that reduced expression of miR-133a was inversely correlated with increased expression of XIST in bladder cancer tissues and that knockdown of XIST caused an upregulation of miR-133a in bladder cancer cells, suggesting that the increased expression of XIST may contribute to the decreased expression of miR-133a in bladder cancer. Furthermore, it was observed that silencing of miR-133a impaired the inhibitory effects of XIST knockdown on the proliferation and migration of bladder cancer cells, indicating that miR-133a is indeed involved in XIST-mediated bladder cancer. In addition to miR-133a, several other miRNAs, including miR-124 (33), miR-139 (22) and miR-200 (21), have also been identified as target miRNAs of XIST in bladder cancer cells. Xiong et al (33) reported that XIST directly interacts with miR-124 and thus promotes the expression of androgen receptor in bladder cancer cells. Hu et al (22) indicated that XIST promoted bladder cancer cell growth and metastasis through interacting with miR-139-5p and thus affected the activity of the Wnt/ $\beta$-catenin signalling pathway. The present study enhances the current understanding of the function of the XIST/miRNA axis in bladder cancer cells. Future studies should focus on identifying the downstream protein targets of miR-133a in bladder cancer.

In conclusion, the present study demonstrated for the first time that XIST promotes bladder cancer cell proliferation and migration via modulation of miR-133a and thus suggests that XIST may be used as a potential therapeutic target for bladder cancer.

\section{Acknowledgements}

Not applicable.

\section{Funding}

No funding was received.

\section{Availability of data and materials}

All data generated or analysed during this study are included in this published article.

\section{Authors' contributions}

$\mathrm{KZ}$ and JY designed the study and wrote the manuscript. WC collected clinical tissues. KZ, XL and WC performed all experiments and performed the statistical analysis.

\section{Ethics approval and consent to participate}

This study was approved by the Ethics Committee of the Second Xiangya Hospital (Changsha, China). Written informed consent was obtained from all patients involved in this study.

\section{Patient consent for publication}

All patients provided written informed consent for their data to be published.

\section{Competing interests}

The authors declare that they have no competing interests.

\section{References}

1. Sathe A and Nawroth R: Targeting the PI3K/AKT/mTOR Pathway in Bladder Cancer. Methods Mol Biol 1655: 335-350, 2018.

2. Torre LA, Bray F, Siegel RL, Ferlay J, Lortet-Tieulent J and Jemal A: Global cancer statistics, 2012. CA Cancer J Clin 65: 87-108, 2015.

3. Siegel RL, Miller KD and Jemal A: Cancer statistics, 2015. CA Cancer J Clin 65: 5-29, 2015.

4. Zhang $X$ and Zhang Y: Bladder cancer and genetic mutations. Cell Biochem Biophys 73: 65-69, 2015.

5. Mao XW, Xiao JQ, Li ZY, Zheng YC and Zhang N: Effects of microRNA-135a on the epithelial-mesenchymal transition, migration and invasion of bladder cancer cells by targeting GSK3 $\beta$ through the Wnt/ $\beta$-catenin signaling pathway. Exp Mol Med 50: e429, 2018.

6. Xie H, Liao X, Chen Z, Fang Y, He A, Zhong Y, Gao Q, Xiao H, Li J, Huang W and Liu Y: LncRNA MALAT1 inhibits apoptosis and promotes invasion by antagonizing miR-125b in bladder cancer cells. J Cancer 8: 3803-3811, 2017.

7. Mitra AP: Molecular substratification of bladder cancer: Moving towards individualized patient management. Ther Adv Urol 8: 215-233, 2016.

8. Smolle MA and Pichler M: The role of long non-coding RNAs in osteosarcoma. Noncoding RNA 4: pii: E7, 2018.

9. Peng Z, Liu C and $\mathrm{Wu} \mathrm{M}$ : New insights into long noncoding RNAs and their roles in glioma. Mol Cancer 17: 61, 2018.

10. Xiong W, Huang C, Deng H, Jian C, Zen C, Ye K, Zhong Z, Zhao X and Zhu L: Oncogenic non-coding RNA NEAT1 promotes the prostate cancer cell growth through the SRC3/IGF1R/AKT pathway. Int J Biochem Cell Biol 94: 125-132, 2018.

11. Cen C, Li J, Liu J, Yang M, Zhang T, Zuo Y, Lin C and Li X: Long noncoding RNA LINC01510 promotes the growth of colorectal cancer cells by modulating MET expression. Cancer Cell Int 18: 45, 2018.

12. Li C, Cui Y, Liu LF, Ren WB, Li QQ, Zhou X, Li YL, Li Y, Bai XY and Zu XB: High expression of long noncoding RNA MALAT1 indicates a poor prognosis and promotes clinical progression and metastasis in bladder cancer. Clin Genitourin Cancer 15: 570-576, 2017.

13. Luo J, Chen J, Li H, Yang Y, Yun H, Yang S and Mao X: LncRNA UCA1 promotes the invasion and EMT of bladder cancer cells by regulating the miR-143/HMGB1 pathway. Oncol Lett 14: 5556-5562, 2017. 
14. Liu D, Li Y, Luo G, Xiao X, Tao D, Wu X, Wang M, Huang C, Wang L, Zeng F and Jiang G: LncRNA SPRY4-IT1 sponges miR-101-3p to promote proliferation and metastasis of bladder cancer cells through up-regulating EZH2. Cancer Lett 388: 281-291, 2017.

15. Zhai X and Xu W: Long noncoding RNA ATB promotes proliferation, migration and invasion in bladder cancer by suppressing microRNA-126. Oncol Res 26: 1063-1072, 2018.

16. Wang M, Guo C, Wang L, Luo G, Huang C, Li Y, Liu D, Zeng F, Jiang $\mathrm{G}$ and Xiao $\mathrm{X}$ : Long noncoding RNA GAS5 promotes bladder cancer cells apoptosis through inhibiting EZH2 transcription. Cell Death Dis 9: 238, 2018.

17. Migeon BR: Choosing the Active $X$ : The human version of $X$ inactivation. Trends Genet 33: 899-909, 2017.

18. Shenoda BB, Tian Y, Alexander GM, Aradillas-Lopez E, Schwartzman RJ and Ajit SK: miR-34a-mediated regulation of XIST in female cells under inflammation. J Pain Res 11: 935-945, 2018.

19. Zhang Q, Chen B, Liu P and Yang J: XIST promotes gastric cancer (GC) progression through TGF- $\beta 1$ via targeting miR-185. J Cell Biochem 119: 2787-2796, 2018.

20. Zhu H, Zheng T, Yu J, Zhou L and Wang L: LncRNA XIST accelerates cervical cancer progression via upregulating Fus through competitively binding with miR-200a. Biomed Pharmacother 105: 789-797, 2018.

21. Xu R, Zhu X, Chen F, Huang C, Ai K, Wu H, Zhang L and Zhao X: LncRNA XIST/miR-200c regulates the stemness properties and tumourigenicity of human bladder cancer stem cell-like cells. Cancer Cell Int 18: 41, 2018.

22. Hu Y, Deng C, Zhang H, Zhang J, Peng B and Hu C: Long non-coding RNA XIST promotes cell growth and metastasis through regulating miR-139-5p mediated Wnt $/ \beta$-catenin signaling pathway in bladder cancer. Oncotarget 8: 94554-94568, 2017.

23. Wei W, Liu Y, Lu Y, Yang B and Tang L: LncRNA XIST promotes pancreatic cancer proliferation through miR-133a/EGFR. J Cell Biochem 118: 3349-3358, 2017.

24. Livak KJ and Schmittgen TD: Analysis of relative gene expression data using real-time quantitative PCR and the 2(-Delta Delta C(T)) method. Methods 25: 402-408, 2001

25. Kruger J and Rehmsmeier M: RNAhybrid: microRNA target prediction easy, fast and flexible. Nucleic Acids Res 34: W451-W454, 2006.

26. Xiao Z, Qu Z, Chen Z, Fang Z, Zhou K, Huang Z, Guo X and Zhang Y: LncRNA HOTAIR is a prognostic biomarker for the proliferation and chemoresistance of colorectal cancer via MiR-203a-3p-mediated Wnt/B-catenin signaling pathway. Cell Physiol Biochem 46: 1275-1285, 2018.
27. Liu K,YaoH,Wen Y,ZhaoH,Zhou N,Lei S and Xiong L:Functional role of a long non-coding RNA LIFR-AS1/miR-29a/TNFAIP3 axis in colorectal cancer resistance to pohotodynamic therapy. Biochim Biophys Acta Mol Basis Dis 1864: 2871-2880, 2018

28. Li C, Wan L, Liu Z, Xu G, Wang S, Su Z, Zhang Y, Zhang C, Liu X, Lei Z and Zhang HT: Long non-coding RNA XIST promotes TGF- $\beta$-induced epithelial-mesenchymal transition by regulating miR-367/141-ZEB2 axis in non-small-cell lung cancer. Cancer Lett 418: 185-195, 2018.

29. Jiang H, Zhang H, Hu X and Li W: Knockdown of long non-coding RNA XIST inhibits cell viability and invasion by regulating miR-137/PXN axis in non-small cell lung cancer. Int J Biol Macromol 111: 623-631, 2018

30. Xu Z, Xu J, Lu H, Lin B, Cai S, Guo J, Zang F and Chen R LARP1 is regulated by the XIST/miR-374a axis and functions as an oncogene in non-small cell lung carcinoma. Oncol Rep 38: 3659-3667, 2017.

31. Yang $\mathrm{C}, \mathrm{Wu} \mathrm{K}$, Wang $\mathrm{S}$ and Wei G: Long non-coding RNA XIST promotes osteosarcoma progression by targeting YAP via miR-195-5p. J Cell Biochem 119: 5646-5656, 2018.

32. Sun Z, Zhang B and Cui T: Long non-coding RNA XIST exerts oncogenic functions in pancreatic cancer via miR-34a-5p. Oncol Rep 39: 1591-1600, 2018.

33. Xiong Y, Wang L, Li Y, Chen M, He W and Qi L: The long non-coding RNA XIST interacted with MiR-124 to modulate bladder cancer growth, invasion and migration by targeting androgen receptor (AR). Cell Physiol Biochem 43: 405-418, 2017.

34. Zhang L, Xu J, Yang G, Li H and Guo X: miR-202 inhibits cell proliferation, migration, and invasion by targeting EGFR in human bladder cancer. Oncol Res 26: 949-957, 2018.

35. Uchida Y, Chiyomaru T, Enokida H, Kawakami K, Tatarano S, Kawahara K, Nishiyama K, Seki N and Nakagawa M: MiR-133a induces apoptosis through direct regulation of GSTP1 in bladder cancer cell lines. Urol Oncol 31: 115-123, 2013.

36. Zhou Y, Wu D, Tao J, Qu P, Zhou Z and Hou J: MicroRNA-133 inhibits cell proliferation, migration and invasion by targeting epidermal growth factor receptor and its downstream effector proteins in bladder cancer. Scand J Urol 47: 423-432, 2013.

This work is licensed under a Creative Commons Attribution-NonCommercial-NoDerivatives 4.0 International (CC BY-NC-ND 4.0) License. 